\title{
Transcriptional and posttranscriptional mechanisms contribute to the dysregulation of elastogenesis in Schimke immuno-osseous dysplasia
}

\author{
Marie Morimoto 1,2 , Karen J. Wang ${ }^{1,2}$, Zhongxin Yu3 , Andrew K. Gormley ${ }^{4}$, David Parham ${ }^{5}$, Radovan Bogdanovic ${ }^{6,7}$, \\ Thomas Lücke ${ }^{8}$, Christy Mayfield ${ }^{9}$, Rosanna Weksberg ${ }^{10}$, Glenda Hendson ${ }^{11,12}$ and Cornelius F. Boerkoel ${ }^{1,2}$
}

BACKGROUND: Schimke immuno-osseous dysplasia (SIOD) is an autosomal recessive disorder caused by mutations in SMARCAL 1. A frequent complication is arteriosclerosis associated with reduced elastin expression; however, the mechanism underlying the reduced elastin expression remains unknown. METHODS: Expression of transcriptional regulators of elastin (ELN) and microRNA (miRNA) regulators of ELN messenger RNA (mRNA), ELN promoter methylation, and ELN mRNA poly(A) tail length were assessed by quantitative RT-PCR, bisulfite Sanger sequencing, and the Poly(A) Tail Length Assay Kit, respectively, in unaffected developing human aortae and in an SIOD aorta. RESULTS: Comparing unaffected fetal and adult aortae, ELN precursor mRNA (pre-mRNA) levels remained nearly constant, whereas mRNA levels declined by $\sim 10^{2}$-fold. This corresponded with a reduction in poly $(A)$ tail length but not with changes in the other parameters. In contrast, compared to the unaffected fetal aortae, the SIOD aorta had 18-fold less ELN pre-mRNA and $10^{4}$-fold less mRNA. This corresponded with increased expression of miRNA regulators and shorter ELN mRNA poly(A) tail lengths but not with altered expression of ELN transcriptional regulators or ELN promoter methylation.

CONCLUSION: Posttranscriptional mechanisms account for the reduction in ELN mRNA levels in unaffected aortae, whereas transcriptional and posttranscriptional mechanisms reduce elastin expression in SIOD aorta and predispose to arteriosclerosis.

$\mathbf{S}$ chimke immuno-osseous dysplasia (SIOD, OMIM 242900) is a rare autosomal recessive multisystemic disorder caused by mutations in the SWI/SNF-related, matrix-associated, actin-dependent regulator of chromatin, subfamily a-like 1 (SMARCAL1) gene (1). SMARCAL1 functions as a DNA-dependent annealing helicase (2) that recognizes single-stranded to double-stranded DNA transitions and binds to transcriptionally active chromatin (3). SMARCAL1 is involved in the DNA stress response $(4,5)$, the reactivation of stalled replication forks (6-8), and the modulation of gene expression (3). Key manifestations of the disease include growth failure due to spondyloepiphyseal dysplasia, renal failure due to focal segmental glomerulosclerosis, T-cell immunodeficiency, facial dysmorphism, and hyperpigmented macules (9). Approximately $50 \%$ of SIOD patients also develop vascular disease (10), and the vascular complications are refractory to treatment and a common cause of death (11).

The vascular disease in SIOD manifests as migraine-like headaches, transient ischemic attacks, and cerebrovascular accidents (11). The arterial histopathology shows intimal and medial hyperplasia, smooth muscle cell hyperplasia, and fragmented and disorganized elastin fibers $(10,12)$. Molecular studies suggest reduced elastogenesis (10); however, the mechanism underlying this remains unknown.

Elastin, which is critical for the development and maintenance of the arteries $(13,14)$, confers the elastic recoil properties required for the proper function of the arteries as well as other load-bearing tissues such as the skin, intestines, and lungs and has signaling capabilities in addition to being a structural protein (14). Mouse models deficient for elastin die shortly after birth due to arterial obstruction, and mouse models haploinsufficient for elastin (Eln) exhibit hypertension and alterations in arterial wall structure (15). Similarly, human haploinsufficiency for elastin (ELN) causes supravalvular aortic stenosis, and elastin deficiency causes lax skin, vascular disease, and respiratory disease (reviewed in 16).

In the aorta of model organisms, elastin protein concentration increases during fetal development to a maximum just following birth and then progressively declines as it is diluted

\footnotetext{
'Department of Medical Genetics, University of British Columbia, Vancouver, British Columbia, Canada; ${ }^{2}$ Child and Family Research Institute, Vancouver, British Columbia, Canada; ${ }^{3}$ Department of Pathology, University of Oklahoma Health Sciences Center, Oklahoma City, Oklahoma; ${ }^{4}$ Department of Pediatrics, University of Oklahoma Health Sciences Center, Oklahoma City, Oklahoma; ${ }^{5}$ Department of Pathology, Children's Hospital Los Angeles, Keck School of Medicine, University of Southern California, Los Angeles, California; ${ }^{6}$ Department of Nephrology, Institute of Mother and Child Healthcare of Serbia, Belgrade, Serbia; ${ }^{7}$ Department of Pediatrics, Faculty of Medicine, University of Belgrade, Belgrade, Serbia; ${ }^{8}$ Department of Neuropediatrics, Children's Hospital, Ruhr-University Bochum, Bochum, Germany; ${ }^{9}$ Warren Clinic, Tulsa, Oklahoma; ${ }^{10}$ Department of

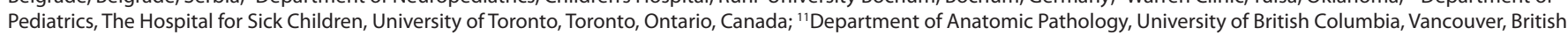
Columbia, Canada; ${ }^{12}$ Department of Anatomic Pathology, Children's and Women's Health Centre of British Columbia, Vancouver, British Columbia, Canada. Correspondence: Cornelius F. Boerkoel (boerkoel@interchange.ubc.ca)
} 
by the addition of other extracellular components (17-19). Similarly, within the human aorta, insoluble elastin protein concentration increases during fetal development (20) and then progressively declines $(21,22)$.

The developmental regulation of elastin production is predominantly posttranscriptional. Based on the study of fetal and postnatal rat lung (23) and fetal and postnatal human skin (24), ELN precursor messenger RNA (pre-mRNA) levels remain stable despite the postnatal decline in steady-state levels of ELN messenger RNA (mRNA). Identified posttranscriptional mechanisms of ELN gene regulation include regulation by microRNAs (miRNAs) (25-27) and poly(A) tail degradation (24).

Although ELN and its regulation have been studied for several decades, its regulation during human aorta development is largely unknown. Therefore, the aim of this study was, first, to establish the transcriptional and posttranscriptional mechanisms that regulate ELN expression in the human aorta during development and, second, to assess these mechanisms in SIOD. The transcriptional mechanisms studied include the expression of transcriptional regulators of ELN and promoter methylation of the ELN gene, as well as the expression of ELN pre-mRNA as a general readout of ELN gene transcription. The posttranscriptional mechanisms studied included the expression of miRNA regulators and poly(A) tail shortening of ELN mRNA. Since elastin expression is vastly different between the fetal and adult aortae, we hypothesized that at least one or more of these mechanisms contributes to the postnatal developmental decrease in ELN mRNA expression and that dysregulation of one or more of these mechanisms contributes to the decreased ELN mRNA expression in SIOD.

\section{RESULTS}

ELN Pre-mRNA Levels, Which Decrease Only Modestly With Aortic Development From Fetus to Adult, Are Markedly Decreased in SIOD

We have previously shown that the relative ELN mRNA level was $\sim 10^{2}$-fold lower in the aorta of a 5 -y-old boy with SIOD than in unaffected adult aortae (10). To determine aortic ELN mRNA expression during development, we performed quantitative RT-PCR (qRT-PCR) and observed a moderate negative correlation between ELN mRNA expression and age $\left(r_{S}=\right.$ $-0.626 ; P=0.004$; Supplementary Figure $\mathbf{S 1}$ online). The relative $E L N$ mRNA level was $10^{2}$-fold lower in adult aortae than in unaffected fetal aortae (Figure 1). In contrast, the relative ELN mRNA level was $10^{4}$-fold lower in the aorta of the 5-y-old boy with SIOD than in unaffected fetal aortae (Figure 1).

To determine the relative contribution of decreased ELN transcription to the decreased ELN mRNA levels in unaffected adult and SIOD aortae, we used intronic primers and qRT-PCR to measure ELN pre-mRNA levels. This showed that adult aortae ELN pre-mRNA was 1.8-fold lower than that of two second-trimester fetal aortae, whereas the 5-y-old male SIOD aorta ELN pre-mRNA was 18-fold lower than the two second-trimester fetal aortae (Figure 2).

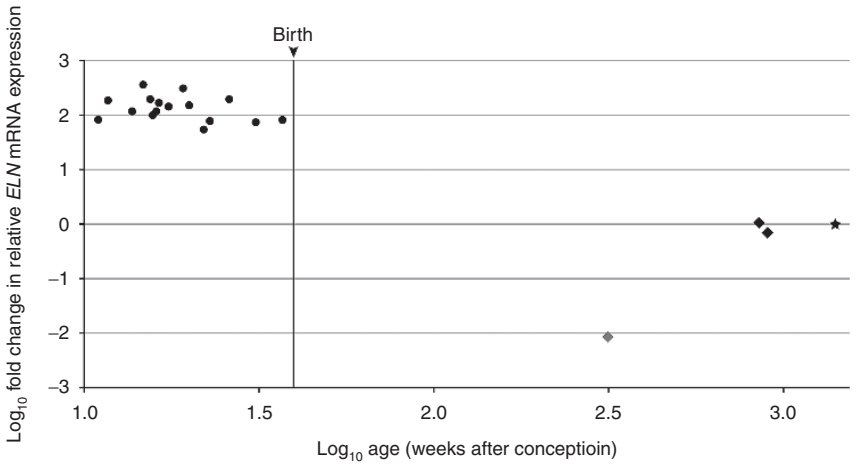

Figure 1. Quantitative PCR of ELN mRNA expression during human aorta development. Expression data are presented as a scatterplot of the $\log _{10}$ fold change in relative $E L N$ mRNA expression in unaffected fetal (black circle) and postnatal (black diamond) human aortae. The gray diamond represents the fold change in ELN mRNA expression in the SIOD aorta as previously published (10). The ELN mRNA levels of three technical replicates were normalized to mRNA levels of the housekeeping gene GAPDH, and the fold changes in ELN mRNA expression were calculated by dividing the normalized ELN mRNA expression levels of the sample of interest by that of the commercially obtained pooled adult human aortae sample (black star). For those fold change values of less than 1, the negative of the reciprocal was calculated as the fold change. The age is represented as the $\log _{10}$ age in weeks after conception with birth (i.e., 40 wk gestation) indicated by the vertical line. SIOD, Schimke immuno-osseous dysplasia.

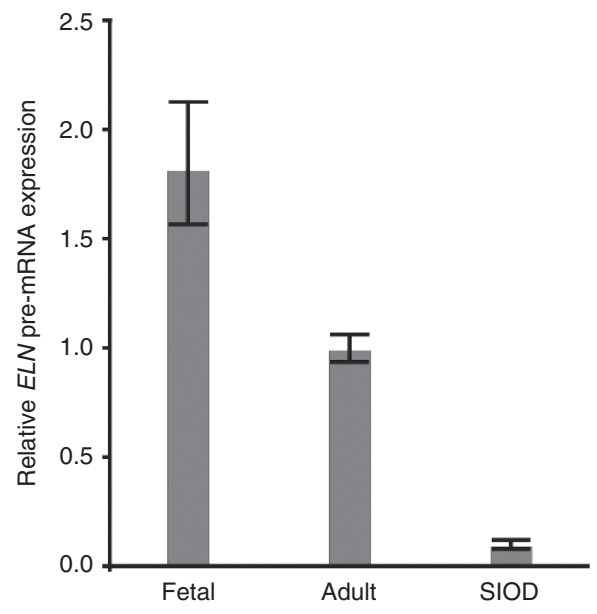

Figure 2. Quantitative PCR of ELN pre-mRNA expression in fetal, adult, and SIOD aortae. Expression data are presented as a bar graph of the relative mean ELN pre-mRNA expression in fetal $(n=2)$, adult $(n=4)$, and SIOD $(n=1)$ aortae. Primers were designed against intron 4 of $E L N$ to assay $E L N$ pre-mRNA expression. The pre-mRNA levels of three technical replicates were normalized to the mRNA levels of the housekeeping gene GAPDH and plotted relative to that of the commercially obtained pooled adult aortae sample. Error bars represent 1 SD. SIOD, Schimke immuno-osseous dysplasia.

SIOD-Associated Alterations in Aortic Expression of Transcriptional Regulators of ELN Parallel Those Observed With Development of the Aorta From Fetus to Adult

Altered expression of transcriptional regulators is associated with the modulation of ELN expression (28-30). We previously observed that the aorta of a 5 -y-old boy with SIOD had altered mRNA levels of some positive and negative transcriptional regulators of $E L N$ (10). To determine if these changes were 
a

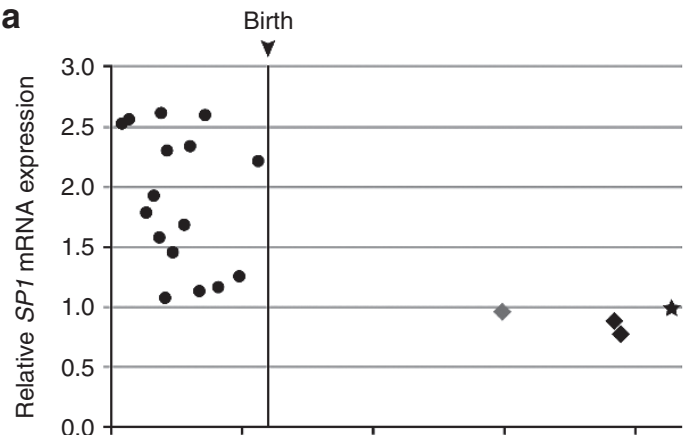

b

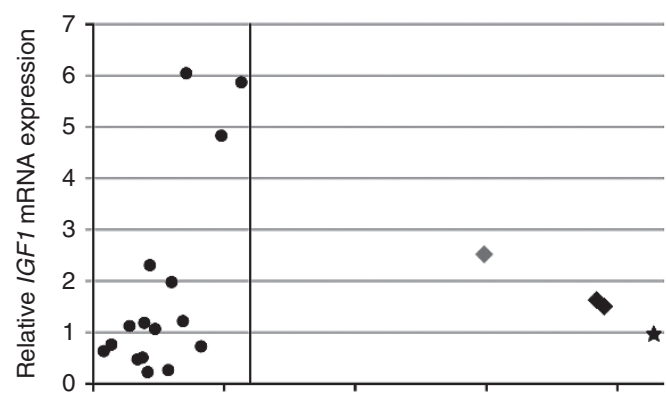

C

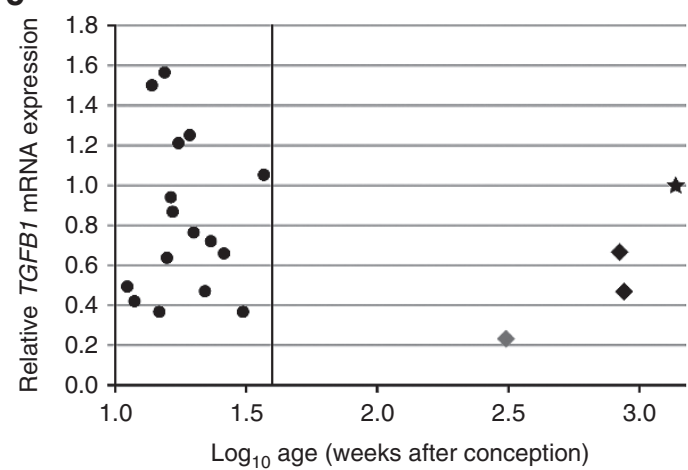

d

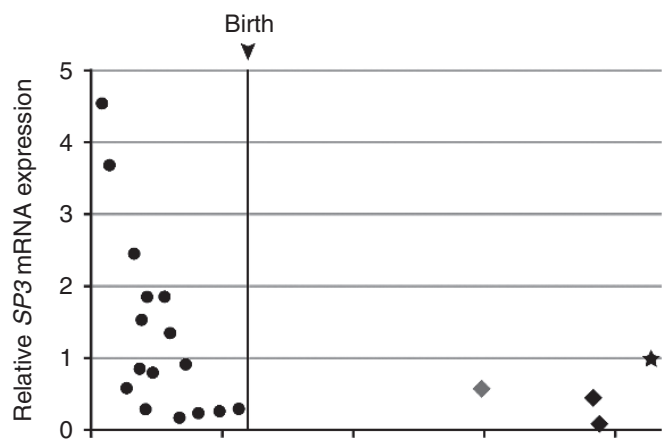

e

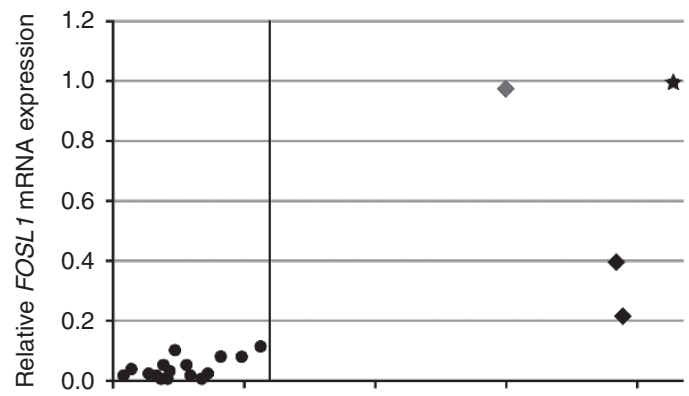

f

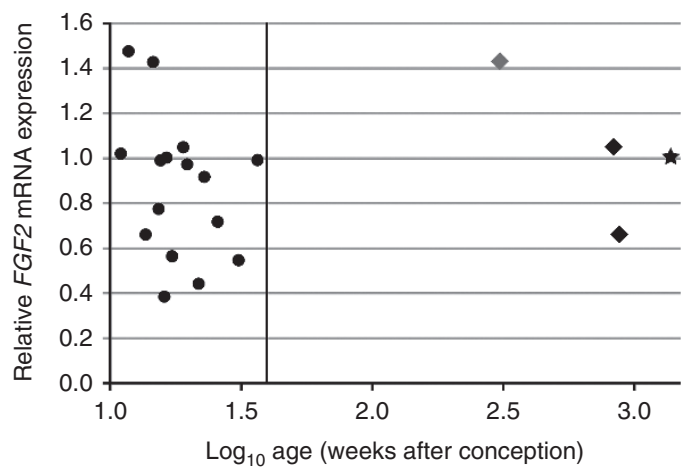

Figure 3. Quantitative PCR of transcriptional regulators of ELN during human aorta development. Positive regulators (a) SP1, (b) IGF1, and (c) TGFB1, and negative regulators (d) SP3, (e) FOSL1, and (f) FGF2 were analyzed and presented as scatterplots of relative mRNA expression in unaffected fetal (black circle) and postnatal (black diamond) human aortae. The gray diamond represents the relative mRNA expression in the SIOD aorta as previously published (10). The mRNA levels of three technical replicates were normalized to the mRNA levels of the housekeeping gene GAPDH and plotted relative to that of the commercially obtained pooled adult human aortae sample (black star). The age is represented as the $\log _{10}$ age in weeks after conception with birth (i.e., 40 wk gestation) indicated by the vertical line. SIOD, Schimke immuno-osseous dysplasia.

peculiar to SIOD or consistent with changes occurring during the development of the aorta from fetus to adult, we profiled the mRNA levels of these transcription factors by qRT-PCR. The transcriptional regulators studied included $S P 1, I G F 1, T G F B 1$, $S P 3, F O S L 1$, and FGF2 (28-30). SP1 mRNA levels declined with development (Figure 3a) and had a moderate negative correlation with age $\left(r_{S}=-0.586 ; P=0.008\right.$; Supplementary Figure $\mathbf{S 1}$ online). IGF1 mRNA levels in the first and second trimesters were comparable to postnatal levels and peaked in the third trimester (Figure 3b). TGFB1 mRNA levels were relatively constant throughout development (Figure 3c) and did not have a significant correlation with age $\left(r_{S}=0.016 ; P=0.949\right.$; Supplementary Figure S1 online). SP3 mRNA levels decreased during development (Figure 3d) and had a moderate negative correlation with age $\left(r_{S}=-0.649 ; P=0.003\right.$; Supplementary Figure S2 online).
FOSL1 mRNA levels increased postnatally (Figure 3e) and had a moderate positive correlation with age $\left(r_{S}=0.647 ; P=0.003\right.$; Supplementary Figure S2 online). Finally, FGF2 mRNA levels were relatively constant throughout development (Figure 3f) and did not have a significant correlation with age $\left(r_{S}=-0.256\right.$; $P=0.290$; Supplementary Figure S2 online). Our previously observed changes in ELN transcriptional regulators within the SIOD aorta are therefore consistent with changes occurring during aortic development and not peculiar to SIOD (Figure 3).

DNA Methylation of the Aortic Proximal ELN Promoter Does Not Change With Development of the Aorta From Fetus to Adult or in SIOD

Often DNA methylation is inversely proportionate to the accessibility of the DNA to transcription factors (reviewed in 


\section{Articles $\mid$ Morimotoetal.}

a

\begin{tabular}{llll} 
chr7:73,440,604 \\
\hline $\mathbf{P}$ PCR fragment 5
\end{tabular}

b
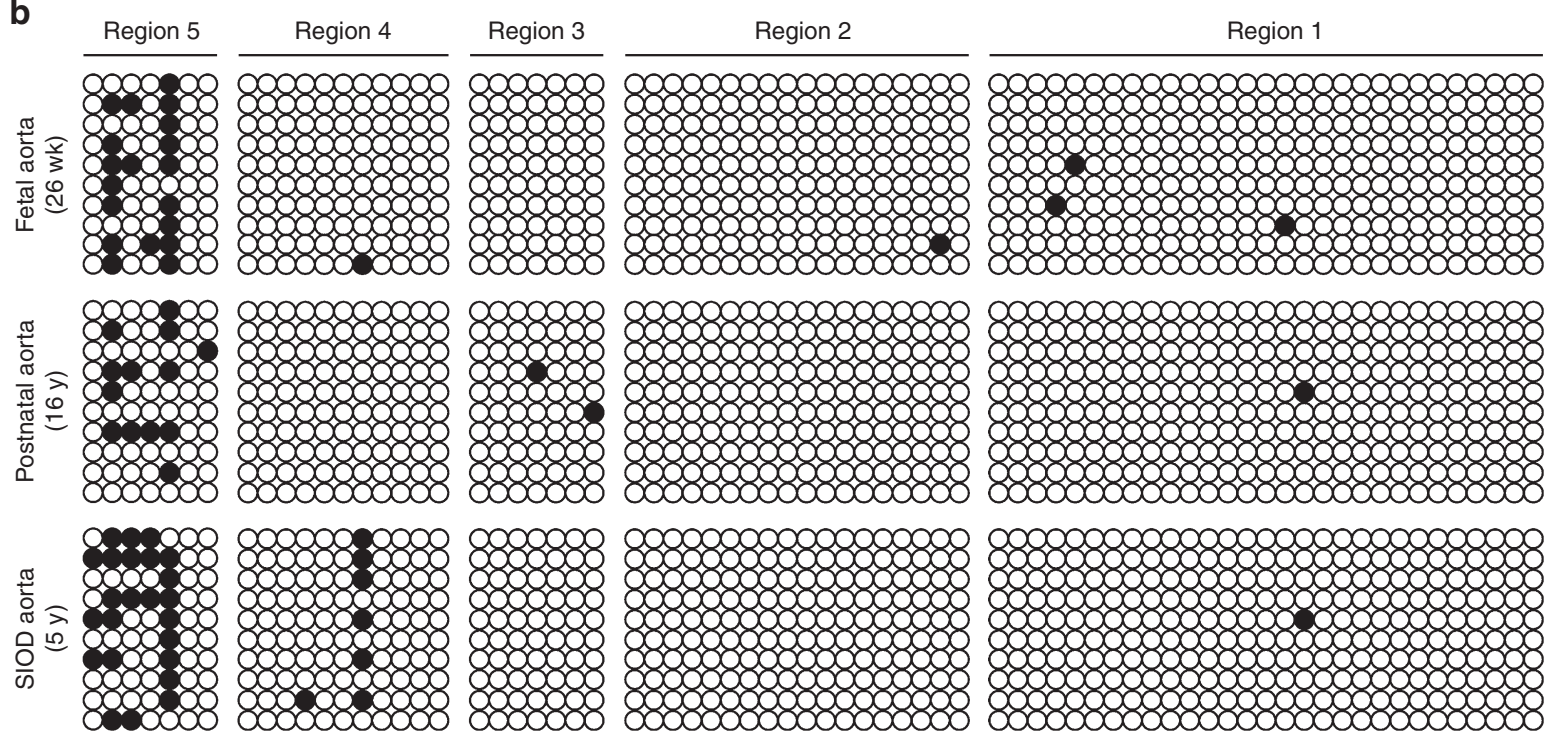

Figure 4. Bisulfite sequencing analysis of the ELN promoter in fetal, postnatal, and SIOD aortae. After bisulfite conversion, the ELN promoter was amplified as five regions and each was cloned and sequenced. (a) A schematic of the PCR fragments and the corresponding CpG sites within each fragment (arrowheads). The location of a CpG island, the first exon (white box) of ELN, and the first intron (black line) of ELN are indicated in the schematic. (b) Methylated $\mathrm{CpGs}$ are represented as black dots and unmethylated $\mathrm{CpGs}$ as white dots. Ten single molecules of each region were sequenced for each sample. Each row represents a single molecule. SIOD, Schimke immuno-osseous dysplasia; TSS, translation start site.

31 , and therefore, promoter methylation frequently reflects transcriptional inhibition. To determine if DNA methylation contributes to the regulation of $E L N$, we assessed the methylation of the aortic ELN promoter ( -1914 to +329 bp relative to the translation start site) of a fetal, postnatal, and SIOD aorta by bisulfite Sanger sequencing. We did not detect increased ELN promoter methylation with development or in the 5-yold male SIOD aorta (Figure 4).

\section{Aortic Expression of miR-29 Family Members Does Not Change With Development of the Aorta From Fetus to Adult but Is Increased in SIOD}

The relative ELN mRNA level in the SIOD aorta is $10^{2}$-fold lower than that in unaffected adult aortae (10); however, the above analyses of $E L N$ transcription account for only part of the reduction in ELN mRNA levels. We hypothesized therefore that posttranscriptional mechanisms also contribute. Increased expression of the conserved miR-29 family has been shown to promote directly the degradation of ELN mRNA in development and disease (25-27). The seed sequence that confers target specificity is identical among all family members, and three conserved miRNA response elements are present in the human ELN 3' untranslated region (3' UTR; Figure 5a) (26,27).

To determine whether there was increased expression of the miR-29 family in development or SIOD, we quantified each family member by qRT-PCR. Comparison of unaffected adult to fetal aortae detected modest changes; miR-29a-3p (accession number: MIMAT0000086) and miR-29c-3p (accession number: MIMAT0000100) levels were respectively increased 4.1-fold and 1.9-fold and miR-29b-3p (accession number: MIMAT0000681) was reduced 1.5 -fold (Figure 5b-d). In contrast, for the 5-y-old SIOD vs. unaffected adult aortae, miR29a-3p, miR-29b-3p, and miR-29c-3p levels were respectively 4-, 45-, and 8-fold higher (Figure $5 \mathbf{b}-\mathbf{d}$ ).

\section{ELN mRNA poly(A) Tail Length in the Aorta Shortens With Development of the Aorta From Fetus to Adult and in SIOD} A marker of posttranscriptional degradation or mRNA instability is a shorter poly(A) tail (reviewed in 32). Prior comparison of ELN mRNA in adult vs. fetal human skin, uterus, and lung showed that the transcripts from adult tissue have shorter poly(A) tails (24). To determine if this also occurred in the human aorta, we compared the mRNA poly(A) tail length of $E L N$ aortic transcripts terminating at the proximal and distal polyadenylation sites and observed that both adult ELN transcripts had shorter poly (A) tails than the respective fetal transcripts (Figure 6). Consistent with the destabilization of ELN mRNA in SIOD, the transcripts extracted from the aorta of the 5-y-old boy with SIOD had a distal poly(A) tail length comparable to that of unaffected adult aortae and a proximal poly(A) tail length shorter than that of unaffected adult aortae (Figure 6). 
a

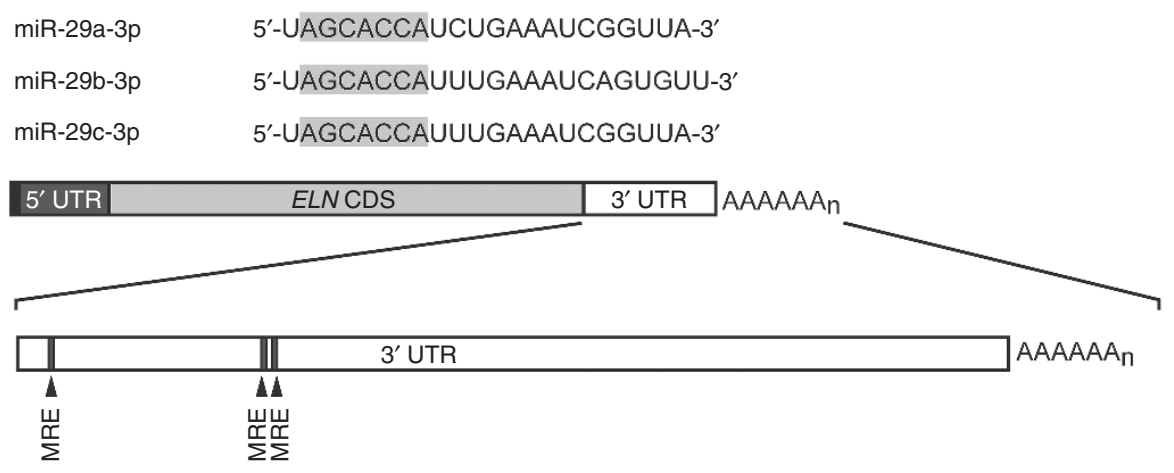

b

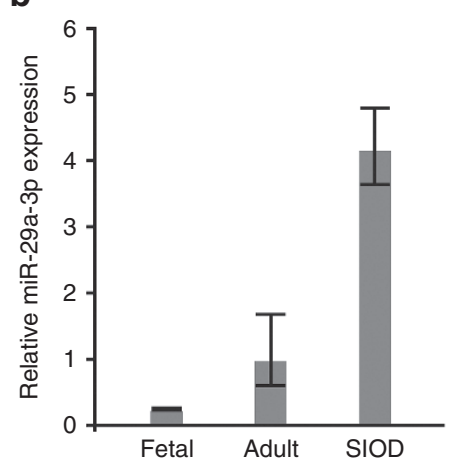

C

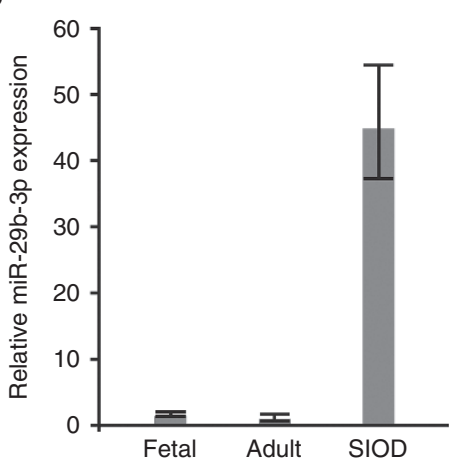

d

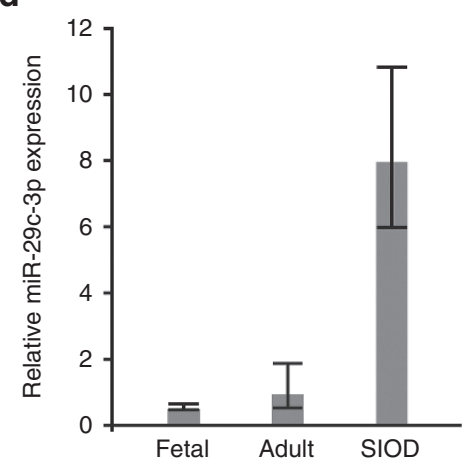

Figure 5. Quantitative PCR of the miR-29 family in fetal, adult, and SIOD aortae. (a) The miRNAs assayed in this study and their identical seed sequences highlighted in gray. The relative positions of the miR-29 miRNA response elements (MREs) are indicated in the schematic. Expression data are presented as bar graphs of the relative mean miRNA expression of $(\mathbf{b})$ miR-29a-3p, (c) miR-29b-3p, and (d) miR-29c-3p in fetal $(n=2)$, adult $(n=4)$, and SIOD $(n=1)$ aortae. The miRNA levels of three technical replicates were normalized to the levels of the small nuclear RNA RNU6-2 and plotted relative to that of the commercially obtained pooled adult aortae sample. Error bars represent 1 SD. SIOD, Schimke immuno-osseous dysplasia.

\section{DISCUSSION}

We have demonstrated that both transcriptional and posttranscriptional mechanisms are associated with reduced elastin expression in an SIOD aorta and shown that this arises, in part, from the dysregulation of known mechanisms for regulating elastin expression. To our knowledge, this is the first study of these elastin regulatory mechanisms in an SIOD aorta and in developing unaffected human aortae.

Prior studies of elastin protein concentration $(21,22)$ and ELN mRNA levels (33) in the human aorta found that both decrease postnatally with age. To define this developmental decline in elastin better, we characterized aortic ELN mRNA expression during fetal development and postnatally. The prenatal expression of ELN mRNA was stable ( $r s=-0.368 ; P=$ 0.161) from late first trimester through the third trimester of gestation. This pattern is consistent with prior histological studies showing initial deposition of extracellular aortic elastin in the latter half of the first trimester (34) and increasing total deposition throughout prenatal development (20). For the fetal aorta, therefore, the increasing deposition and concentration of insoluble elastin arises from the accumulation of elastin protein and the continued high expression of ELN mRNA.

Compared to the levels of aortic ELN mRNA during fetal development, we found markedly lower levels of ELN mRNA in the postnatal aorta. Similar to findings in other tissues (24), our quantification of ELN pre-mRNA in human fetal and adult aortae demonstrated only a 1.8 -fold decrease in adult
ELN transcription. Consistent with this steady transcription of $E L N$, we did not detect marked changes in the mRNA levels of known ELN transcriptional regulators or in methylation of the proximal ELN promoter. We conclude, therefore, that as in other tissues, the postnatal reduction in aortic ELN mRNA levels arises via posttranscriptional mechanisms.

Both miRNAs and a shortened poly $(\mathrm{A})$ tail contribute to the decreased postnatal levels of ELN mRNA in other tissues or organisms (reviewed in 35). In contrast to mice (26), we did not observe a marked increase in the miR-29 family of miRNAs in adult human aortae; this might reflect a species-specific difference or be a result of the number of samples or time points we tested. On the other hand, consistent with increased ELN mRNA degradation, both the proximal and distal poly(A) tails of ELN mRNA were shorter in adult aortae than that in fetal aortae; this agrees with findings in other elastin-rich human tissues (24) and affirms decreased ELN mRNA stability as a factor underlying the reduced postnatal levels of ELN mRNA in the aorta.

In contrast to ELN mRNA regulation during the development of unaffected aortae, both transcriptional and posttranscriptional mechanisms contribute to the markedly decreased levels of ELN mRNA in the SIOD aorta. The markedly decreased $E L N$ pre-mRNA levels define a reduction in ELN transcription that is not attributable to the altered expression of known ELN transcriptional regulators or to the methylation of the proximal ELN promoter; consequently, other transcriptional regulatory 
a

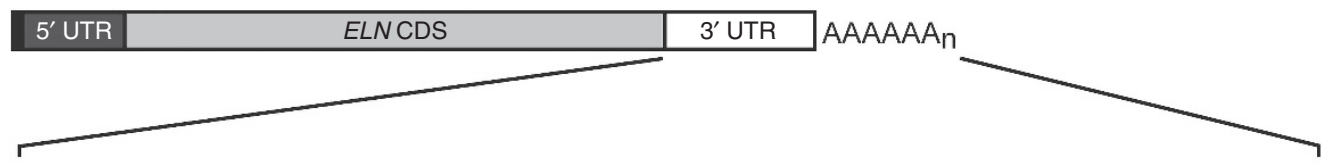

Proximal polyadenylation site transcript

\begin{tabular}{|l|||c|}
\hline & $3^{\prime}$ UTR \\
\hline
\end{tabular}

Distal polyadenylation site transcript

b
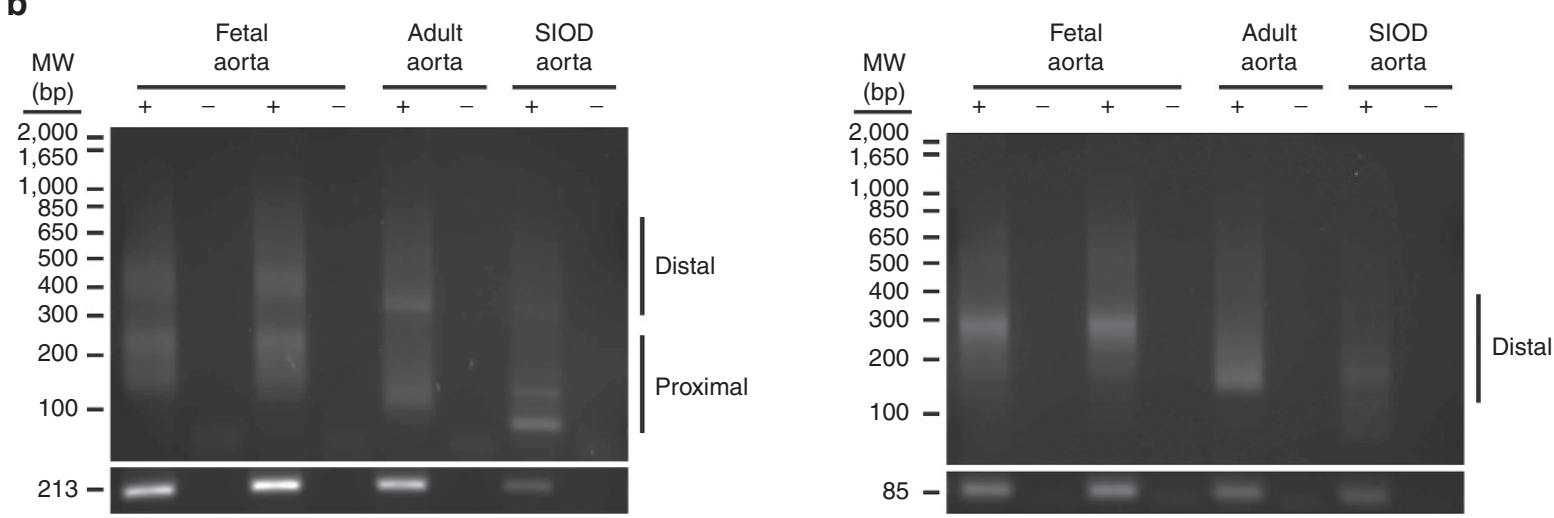

Figure 6. Poly(A) tail length analysis of ELN mRNA in fetal, adult, and SIOD aortae. (a) A schematic of the primer design for the analysis of ELN mRNA transcripts terminating at its two polyadenylation sites. The forward primer I and universal reverse primer assay transcripts terminating at both the proximal and distal polyadenylation sites, whereas the forward primer II and universal reverse primer assay transcripts terminating at only the distal polyadenylation site. A gene-specific control PCR with each of the forward primers and corresponding reverse primers was conducted to ensure that the forward primer specifically bound the ELN mRNA 3' UTR. The relative positions of the miR-29 miRNA response elements (MREs) are also indicated in the schematic. (b) Poly(A) tail products (top) and gene-specific controls (bottom) for the proximal and distal (left) and distal only (right) poly(A) tail assays. +, reverse transcription; -, no reverse transcription negative control; bp, base pairs; CDS, coding DNA sequence; MW, molecular weight; SIOD, Schimke immuno-osseous dysplasia; 3' UTR, 3' untranslated region.

mechanisms such as chromatin accessibility, histone modifications, and additional transcription factors should be tested. Regarding posttranscriptional mechanisms, both increased miR-29a-3p, miR-29b-3p, and miR-29c-3p expression and accelerated poly $(\mathrm{A})$ tail shortening appear to contribute to the reduced levels of ELN mRNA in the SIOD aorta. Previous gene expression studies of the same SIOD aorta compared to a pooled unaffected adult aortae sample showed that other genes targeted by miR-29 in human elastin-rich tissues were not substantially altered, such as COL1A1 (fold change $=1.39$; $P=0.019$ ) and $C O L 3 A 1$ (fold change $=1.35 ; P=0.0017$ ), but other genes were decreased as expected, such as VEGFA (fold change $=-2.48 ; P=0.019)(10)$; these results highlight the complexity of miR-29 regulation of mRNA levels and the ambiguity of a causal association with the reduced ELN mRNA levels. Though there was no apparent accelerated poly(A) tail shortening of the distal polyadenylation site transcript, there was substantial paucity of the transcript. This observed paucity, however, was not due to the increased miR-29 expression since all three miR-29 miRNA response elements are present in both proximal and distal polyadenylation site transcripts (Figure 6a).

Comparison of the SIOD aorta to a developmental series of human aortae establishes the validity of our assays and allows us to hypothesize whether the processes underlying the reduced levels of ELN mRNA in SIOD aorta are dysregulated developmental mechanisms or pathologic mechanisms unique to SIOD. The reduced transcription of ELN and the increased expression of the miR-29 regulators of ELN mRNA are pathologic processes unique to SIOD. In contrast, the more severe shortening of the poly $(\mathrm{A})$ tails suggests dysregulation of a developmental process for regulating aortic ELN mRNA levels.

The deficiency of the multifunctional SMARCAL1 annealing helicase could lead to the ELN gene dysregulation in the aorta 
in the following ways. SMARCAL1 could alter the local structure of DNA thereby promoting or inhibiting the transcription of specific genes via a direct effect of SMARCAL1 deficiency on the gene of interest (e.g., ELN) or from an indirect effect of SMARCAL1 deficiency on upstream pathways (e.g., increased expression of miR-29 family members, altered expression of the pathway regulating ELN poly(A) tail length, etc.). We hypothesize that SMARCAL1 deficiency mediates these effects by altering the epigenetic landscape as a consequence of its role in the DNA damage response $(4,5)$ and replication fork restart $(6-8)$. Replication fork stalling is associated with epigenetic instability (36); consequently, if SMARCAL1 replication fork stalling occurred near ELN or key regulators of ELN and ELN mRNA stability, the resulting epigenetic changes might produce the pathogenic gene expression alterations observed in the SIOD aorta.

In the context of dissecting the mechanisms by which SMARCAL1 deficiency alters transcriptional and posttranscriptional mechanisms of ELN mRNA regulation, we evaluated cultured skin fibroblasts and knockout mice as model systems. Although cultured dermal fibroblasts are frequently used as a model for elastogenesis $(37,38)$, cultured primary dermal fibroblasts of SIOD patients did not consistently manifest markedly lower ELN mRNA levels compared to agematched controls (Supplementary Figure S3 online). Also, the ELN mRNA levels did not correlate with whether the SIOD patient had vascular or lung disease. Similarly, knockout mice showed no consistent difference in ELN mRNA levels (data not shown). The Smarcal1 knockout mouse might not have manifested vascular disease or marked dysregulated elastogenesis for several reasons. First, SMARCAL1 homologues are proteins that recognize DNA structure rather than DNA sequence, and the altered chromatin landscape in mice might lead to different gene targets of Smarcal1 deficiency and therefore not lead to Eln gene dysregulation. Second, humans are exquisitely prone to vascular disease and sensitive to elastin deficiency, whereas wild-type mice are resistant to vascular disease and only develop vascular disease with defined genetic modifications $(39,40)$. These biological differences might explain why we were unable to detect a molecular phenotype in the mice. The cultured primary dermal fibroblasts of SIOD patients also did not exhibit dysregulated elastogenesis, which we hypothesize is secondary to cell type-related differences since not all tissues are equally affected in SIOD; for example, patients do not have any pronounced skin manifestations, other than hyperpigmented macules (11).

Key limitations of this study are the modest number of samples analyzed and the absence of age-matched samples for these analyses of $E L N$ gene expression and regulation. Human aorta is a difficult tissue to collect, and SIOD is a rare disorder. However, we performed a number of hypothesis-driven targeted studies to utilize this limited resource fully and to provide as much insight as possible into the dysregulation of $E L N$ in SIOD as well as the regulation of ELN in normal aorta development. Further studies on additional SIOD samples are required to verify these findings.

\section{METHODS}

\section{Patients}

The guardians of the patient referred to this study signed informed consent documents approved by the University of British Columbia (Vancouver, Canada). Autopsy tissues were obtained according to the protocol approved by the University of British Columbia. SD120 was a 5-y-old male SIOD patient who has been previously described (10).

\section{Human Tissues}

In accordance with institutional policies as approved by the Institutional Review Board (41557) at the University of Washington, human fetal aortae from first- and second-trimester elective terminations were provided as de-identified specimens by the Laboratory of Developmental Biology at the University of Washington (Seattle, WA), a National Institute of Child Health \& Human Development supported program. Human postmortem aortae from second- and third-trimester fetuses and a 16- and 17-y-old female were provided as de-identified specimens according to protocol H06-70283 approved by the Clinical Research Ethics Board at the University of British Columbia. The study was carried out according to The Code of Ethics of the World Medical Association (Declaration of Helsinki). Human adult aortae total RNA pooled from four unaffected individuals ranging in age from 27 to $45 \mathrm{y}$ was purchased from Clontech (Mountain View, CA). Sample characteristics and sample usage with respect to each figure are noted in Supplementary Table S1 online.

Dermal fibroblasts from SIOD patients were isolated and cultured from skin biopsies of the forearm. The SMARCAL1 mutations of the SIOD patients from which the dermal fibroblasts were isolated are listed in Supplementary Table S2 online. The following unaffected control dermal fibroblast cell lines were obtained from the Coriell Institute for Medical Research (Camden, NJ): GM00942 (from a 5-yold Caucasian female), GM003348 (from a 9-y-old Caucasian male), and GM05980 (from a 15-y-old Caucasian female).

\section{RNA Extraction and Reverse Transcription}

For the pre-mRNA and mRNA expression studies, total RNA was extracted from flash frozen aorta pulverized with a Bessman tissue pulverizer (Spectrum Laboratories, Rancho Dominguez, CA) and subsequently processed using the RNeasy Mini Kit (Qiagen, Toronto, Canada) according to the manufacturer's specifications. For the cultured dermal fibroblast cells, total RNA was extracted using the RNeasy Mini Kit (Qiagen). Residual genomic DNA was removed by on-column DNase I digestion (Qiagen). Reverse transcription was performed with the qScript cDNA SuperMix (Quanta Biosciences, Gaithersburg, MD) according to the manufacturer's specifications.

For the miRNA expression studies, total RNA including miRNA and other small RNAs was extracted from flash frozen aorta pulverized with a Bessman tissue pulverizer (Spectrum Laboratories) using the miRNeasy Mini Kit (Qiagen) according to the manufacturer's specifications.

\section{Quantitative PCR}

To analyze the expression of ELN mRNA and pre-mRNA as well as known transcriptional regulators of ELN, SsoFast EvaGreen Supermix (Bio-rad Laboratories, Mississauga, Canada) was used with the StepOnePlus Real-Time PCR System (Applied Biosystems by Life Technologies, Burlington, Canada). Expression of the housekeeping gene GAPDH was used as an endogenous control. The primer sequences are listed in Supplementary Table S3 online. Expression analyses were performed with the StepOnePlus software version 2.1 (Applied Biosystems by Life Technologies).

\section{Bisulfite Sanger Sequencing}

Genomic DNA was extracted from the aorta samples, and $500 \mathrm{ng}$ of DNA was bisulfite converted using the EZ DNA Methylation-Gold Kit (Zymo Research, Irvine, CA) according to the manufacturer's protocol. Five overlapping regions of the ELN promoter were amplified by PCR with the HotStarTaq Master Mix Kit (Qiagen) and subsequently cloned into the pCR2.1 vector using The Original TA Cloning Kit (Invitrogen by Life Technologies, Carlsbad, CA). A schematic of the five amplified regions is presented in Figure 4a. The primer sequences used to amplify the five regions were designed using the 
MethPrimer program (http://www.urogene.org/methprimer/) and are listed in Supplementary Table S4 online. Individual clones were isolated and PCR amplified with the following primers: pCR2.1-F (5'-ATGACCATGATTACGCCAAG-3') and pCR2.1-R (5'-CGACTCACTATAGGGCGAATTG-3'). The PCR products were sequenced using Sanger sequencing; for each sample, 10 clones of each fragment were analyzed. The sequence data were analyzed using the Sequencher version 5.1 software (Genecodes, Ann Arbor, MI).

\section{miR-29 Expression Analysis}

To analyze the expression of the miR-29 family of miRNAs in the aorta, we used the miScript PCR System (Qiagen) according to the manufacturer's protocol. Briefly, $135 \mathrm{ng}$ of total RNA was reverse transcribed using the HiSpec Buffer in a $10 \mu \mathrm{l}$ reaction. The cDNA was diluted 1:5, and $1 \mu \mathrm{l}$ of the diluted cDNA was used per $10 \mu \mathrm{l}$ reaction. All quantitative PCRs were performed in triplicate using the QuantiTect SYBR Green PCR Master Mix (Qiagen) and the miScript Primer Assays Hs_miR-29a_1, Hs_miR-29b_1, and Hs_miR-29c_1 (forward primers) (Qiagen) and the miScript Universal Primer (reverse primer). Results were normalized to the level of small nuclear RNA RNU6-2. Quantitative PCR was performed on the StepOnePlus Real-Time PCR System (Applied Biosystems by Life Technologies). Expression analyses were performed with the StepOnePlus software version 2.1 (Applied Biosystems by Life Technologies).

\section{Poly(A) Tail Length Assay}

To determine the poly(A) tail length of ELN mRNA, the Poly(A) Tail Length Assay Kit (USB/Affymetrix, Cleveland, $\mathrm{OH}$ ) was used according to the manufacturer's protocol. In brief, $250 \mathrm{ng}$ of DNase I-treated RNA was used for the initial tailing reaction. Five microliters of each tailed RNA sample was used for reverse transcription. The cDNA was then diluted 1:1 with water and $2 \mu \mathrm{l}$ was used for each PCR. ELN mRNA has two poly(A) sites as previously described (24). To amplify the ELN mRNA poly(A) tails, the universal primer supplied by the manufacturer and forward primer I or forward primer II were used to amplify transcripts terminating at the proximal and distal or only distal polyadenylation site(s), respectively. To ensure that the forward primers were specifically binding to the ELN mRNA 3' UTR, a genespecific control PCR was performed using the primer I pair and the primer II pair (Figure 6a and Supplementary Table S5 online). DNA fragments were separated on a $2 \%$ tris-acetate EDTA agarose gel and visualized by ethidium bromide staining. The $1 \mathrm{~Kb}$ Plus DNA Ladder (Invitrogen) was used as a molecular weight marker.

\section{Cell Culture}

Dermal fibroblasts from SIOD patients and unaffected age-matched individuals were cultured in high glucose Dulbecco's Modified Eagle Medium (DMEM, Gibco/Life Technologies) supplemented with 15\% fetal bovine serum (Gibco/Life Technologies) and $1 \times$ AntibioticAntimycotic (Gibco/Life Technologies) at $37^{\circ} \mathrm{C}$ and $5 \% \mathrm{CO}_{2}$.

\section{Statistical Analyses}

To evaluate the correlation between age and the expression of ELN mRNA and known transcriptional regulators of ELN, the nonparametric Spearman's rank order correlation was used. Statistical significance was defined as a $P$ value of less than 0.05 for a one-tailed test (ELN mRNA expression vs. age) or a two-tailed test (transcription factor expression vs. age). Statistical analyses were conducted using SPSS Statistics version 21 (IBM, Armonk, NY).

\section{SUPPLEMENTARY MATERIAL}

Supplementary material is linked to the online version of the paper at http:// www.nature.com/pr

\section{ACKNOWLEDGMENTS}

We are grateful to the patient and his family members who have contributed to this study. We thank Armin Mario Pühringer and Matthew Lorincz for their technical support with the bisulfite Sanger sequencing. We thank Alireza Baradaran-Heravi for critical review of the manuscript.

\section{STATEMENT OF FINANCIAL SUPPORT}

This work was supported in part by the New Investigator Award jointly sponsored by the SickKids Foundation (Toronto, Canada) and the Canadian
Institutes of Health Research: Institute of Human Development, Child and Youth Health (Ottawa, Canada) (XG09-025 to C.F.B.), and grants from the Michael Smith Foundation for Health Research (Vancouver, Canada) $(\mathrm{Cl}-$ SCH-O1899(07-1) to C.F.B.), The Little Giants Foundation (Gretna, NE) (C.F.B.), and Asociacion Española de Displasias Oseas Minoritarias (Cartagena, Spain) (C.F.B.). Human fetal tissue was obtained through the Laboratory of Developmental Biology project supported by National Institutes of Health Award Number 5R24HD000836 from the Eunice Kennedy Shriver National Institute of Child Health \& Human Development (Bethesda, MD). M.M. was supported by a Four-Year Doctoral Fellowship from the University of British Columbia (Vancouver, Canada). K.J.W. was supported by a Summer Studentship from the Child \& Family Research Institute (Vancouver, Canada). C.F.B. is a scholar of the Michael Smith Foundation for Health Research (Vancouver, Canada) and a Clinical Investigator of the Child \& Family Research Institute (Vancouver, Canada).

Disclosure: The authors declare that there are no conflicts of interest regarding the publication of this article.

\section{REFERENCES}

1. Boerkoel CF, Takashima H, John J, et al. Mutant chromatin remodeling protein SMARCAL1 causes Schimke immuno-osseous dysplasia. Nat Genet 2002;30:215-20.

2. Yusufzai T, Kadonaga JT. HARP is an ATP-driven annealing helicase. Science 2008;322:748-50.

3. Baradaran-Heravi A, Cho KS, Tolhuis B, et al. Penetrance of biallelic SMARCAL1 mutations is associated with environmental and genetic disturbances of gene expression. Hum Mol Genet 2012;21:2572-87.

4. Postow L, Woo EM, Chait BT, Funabiki H. Identification of SMARCAL1 as a component of the DNA damage response. J Biol Chem 2009;284: 35951-61.

5. Yusufzai T, Kong X, Yokomori K, Kadonaga JT. The annealing helicase HARP is recruited to DNA repair sites via an interaction with RPA. Genes Dev 2009;23:2400-4.

6. Bansbach CE, Bétous R, Lovejoy CA, Glick GG, Cortez D. The annealing helicase SMARCAL1 maintains genome integrity at stalled replication forks. Genes Dev 2009;23:2405-14.

7. Ciccia A, Bredemeyer AL, Sowa ME, et al. The SIOD disorder protein SMARCAL1 is an RPA-interacting protein involved in replication fork restart. Genes Dev 2009;23:2415-25.

8. Yuan J, Ghosal G, Chen J. The annealing helicase HARP protects stalled replication forks. Genes Dev 2009;23:2394-9.

9. Schimke RN, Horton WA, King CR. Chondroitin-6-sulphaturia, defective cellular immunity, and nephrotic syndrome. Lancet 1971;2:1088-9.

10. Morimoto M, Yu Z, Stenzel P, et al. Reduced elastogenesis: a clue to the arteriosclerosis and emphysematous changes in Schimke immuno-osseous dysplasia? Orphanet J Rare Dis 2012;7:70.

11. Boerkoel CF, O’Neill S, André JL, et al. Manifestations and treatment of Schimke immuno-osseous dysplasia: 14 new cases and a review of the literature. Eur J Pediatr 2000;159:1-7.

12. Clewing JM, Antalfy BC, Lücke T, et al. Schimke immuno-osseous dysplasia: a clinicopathological correlation. J Med Genet 2007;44:122-30.

13. Li DY, Brooke B, Davis EC, et al. Elastin is an essential determinant of arterial morphogenesis. Nature 1998;393:276-80.

14. Karnik SK, Brooke BS, Bayes-Genis A, et al. A critical role for elastin signaling in vascular morphogenesis and disease. Development 2003;130: 411-23.

15. Faury G, Pezet M, Knutsen RH, et al. Developmental adaptation of the mouse cardiovascular system to elastin haploinsufficiency. J Clin Invest 2003;112:1419-28.

16. Milewicz DM, Urbán Z, Boyd C. Genetic disorders of the elastic fiber system. Matrix Biol 2000;19:471-80.

17. Looker T, Berry CL. The growth and development of the rat aorta. II. Changes in nucleic acid and scleroprotein content. J Anat 1972;113: $17-34$.

18. McCloskey DI, Cleary EG. Chemical composition of the rabbit aorta during development. Circ Res 1974;34:828-35.

19. Davidson JM, Hill KE, Alford JL. Developmental changes in collagen and elastin biosynthesis in the porcine aorta. Dev Biol 1986;118:103-11. 
20. Berry CL, Looker T, Germain J. Nucleic acid and scleroprotein content of the developing human aorta. J Pathol 1972;108:265-74.

21. Myers VC, Lang WW. Some chemical changes in the human thoracic aorta accompanying the aging process. J Gerontol 1946;1:441-4.

22. Andreotti L, Bussotti A, Cammelli D, et al. Aortic connective tissue in ageing-a biochemical study. Angiology 1985;36:872-9.

23. Swee MH, Parks WC, Pierce RA. Developmental regulation of elastin production. Expression of tropoelastin pre-mRNA persists after downregulation of steady-state mRNA levels. J Biol Chem 1995;270:14899-906.

24. Hagmeister U, Reuschlein K, März A, et al. Poly(A) tail shortening correlates with mRNA repression in tropoelastin regulation. J Dermatol Sci 2012;67:44-50.

25. van Rooij E, Sutherland LB, Thatcher JE, et al. Dysregulation of microRNAs after myocardial infarction reveals a role of miR-29 in cardiac fibrosis. Proc Natl Acad Sci USA 2008;105:13027-32.

26. Ott CE, Grünhagen J, Jäger M, et al. MicroRNAs differentially expressed in postnatal aortic development downregulate elastin via 3' UTR and codingsequence binding sites. PLoS One 2011;6:e16250.

27. Zhang P, Huang A, Ferruzzi J, et al. Inhibition of microRNA-29 enhances elastin levels in cells haploinsufficient for elastin and in bioengineered vessels-brief report. Arterioscler Thromb Vasc Biol 2012;32:756-9.

28. Conn KJ, Rich CB, Jensen DE, et al. Insulin-like growth factor-I regulates transcription of the elastin gene through a putative retinoblastoma control element. A role for Sp3 acting as a repressor of elastin gene transcription. J Biol Chem 1996;271:28853-60.

29. Carreras I, Rich CB, Panchenko MP, Foster JA. Basic fibroblast growth factor decreases elastin gene transcription in aortic smooth muscle cells. J Cell Biochem 2002;85:592-600.

30. Kuang PP, Zhang XH, Rich CB, Foster JA, Subramanian M, Goldstein RH. Activation of elastin transcription by transforming growth fac- tor-beta in human lung fibroblasts. Am J Physiol Lung Cell Mol Physiol 2007;292:L944-52.

31. Jaenisch R, Bird A. Epigenetic regulation of gene expression: how the genome integrates intrinsic and environmental signals. Nat Genet 2003;33 Suppl:245-54.

32. Wilusz CJ, Wormington M, Peltz SW. The cap-to-tail guide to mRNA turnover. Nat Rev Mol Cell Biol 2001;2:237-46.

33. Fritze O, Romero B, Schleicher M, et al. Age-related changes in the elastic tissue of the human aorta. J Vasc Res 2012;49:77-86.

34. Frankel HH, Bernick S, Patek PR, Edmondson HA, Peters RL, Paule WJ. Elastic membranes of the developing human aorta. A histochemical and morphological investigation. Arch Pathol 1963;76:474-83.

35. Chen K, Rajewsky N. The evolution of gene regulation by transcription factors and microRNAs. Nat Rev Genet 2007;8:93-103.

36. Schiavone D, Guilbaud G, Murat P, et al. Determinants of G quadruplex-induced epigenetic instability in REV1-deficient cells. EMBO J 2014;33:2507-20.

37. Giro MG, Oikarinen AI, Oikarinen H, Sephel G, Uitto J, Davidson JM. Demonstration of elastin gene expression in human skin fibroblast cultures and reduced tropoelastin production by cells from a patient with atrophoderma. J Clin Invest 1985;75:672-8.

38. Sephel GC, Davidson JM. Elastin production in human skin fibroblast cultures and its decline with age. J Invest Dermatol 1986;86:279-85.

39. Powell-Braxton L, Véniant $M$, Latvala RD, et al. A mouse model of human familial hypercholesterolemia: markedly elevated low density lipoprotein cholesterol levels and severe atherosclerosis on a low-fat chow diet. Nat Med 1998;4:934-8.

40. Zhang SH, Reddick RL, Piedrahita JA, Maeda N. Spontaneous hypercholesterolemia and arterial lesions in mice lacking apolipoprotein E. Science 1992;258:468-71. 